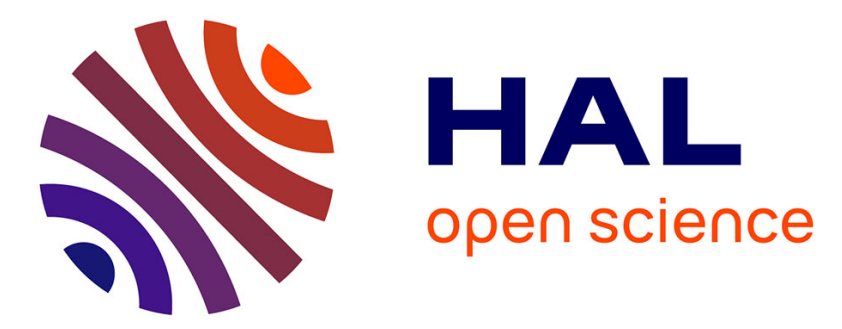

\title{
The social value of information and the competition motive: Price vs. quantity games
}

Camille Cornand, Rodolphe dos Santos Ferreira

\section{To cite this version:}

Camille Cornand, Rodolphe dos Santos Ferreira. The social value of information and the competition motive: Price vs. quantity games . 2017. halshs-01614815v2

\section{HAL Id: halshs-01614815 \\ https://shs.hal.science/halshs-01614815v2}

Preprint submitted on 23 Apr 2018

HAL is a multi-disciplinary open access archive for the deposit and dissemination of scientific research documents, whether they are published or not. The documents may come from teaching and research institutions in France or abroad, or from public or private research centers.
L'archive ouverte pluridisciplinaire HAL, est destinée au dépôt et à la diffusion de documents scientifiques de niveau recherche, publiés ou non, émanant des établissements d'enseignement et de recherche français ou étrangers, des laboratoires publics ou privés. 
UMR 5824

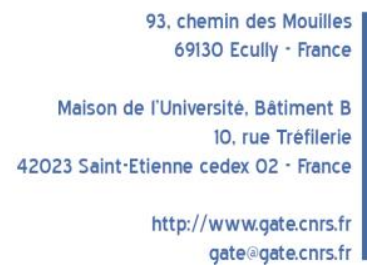

WP 1727 - October 2017, revised April 2018

\title{
The social value of information and the competition motive: Price vs. quantity games \\ Camille Cornand, Rodolphe Dos Santos Ferreira
}

\begin{abstract}
:
We propose a unified framework bridging the gap between team and competition issues, in order to reconsider the social value of private and public information in price and quantity games, when information is both imperfect and dispersed, and to compare the corresponding outcomes in terms of equilibrium and social welfare. In addition to the well-known strategy distortion (from the point of view of the team problem), the competition motive introduces an informational distortion which may lead to a negative social value of private information and reverse the perfect information result in favor of strategic substitutability as the source of higher profit and social welfare
\end{abstract}

\section{Keywords:}

beauty contest, competition, coordination, strategic complementarity, anti-coordination, strategic substitutability, price game, quantity game, dispersed information, public information

JEL codes:

D43, D82, L13

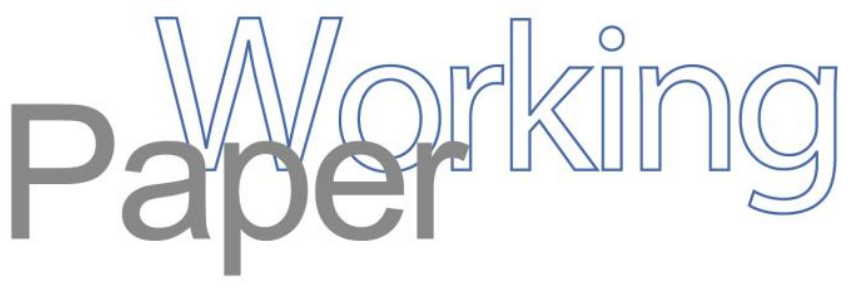




\title{
The social value of information and the competition motive: Price vs. quantity games*
}

\author{
Camille Cornand ${ }^{\dagger}$ \\ Rodolphe Dos Santos Ferreira ${ }^{\ddagger}$
}

April 20, 2018

\begin{abstract}
We propose a unified framework bridging the gap between team and competition issues, in order to reconsider the social value of private and public information in price and quantity games, when information is both imperfect and dispersed, and to compare the corresponding outcomes in terms of equilibrium and social welfare. In addition to the well-known strategy distortion (from the point of view of the team problem), the competition motive introduces an informational distortion which may lead to a negative social value of private information and reverse the perfect information result in favor of strategic substitutability as the source of higher profit and social welfare.
\end{abstract}

Keywords: beauty contest, competition, coordination, strategic complementarity, anti-coordination, strategic substitutability, price game, quantity game, dispersed information, public information.

JEL codes: D43, D82, L13.

\section{Introduction}

The payoffs of Keynes' beauty contest, as modelled by Morris and Shin (2002), involve three motives (Cornand and Heinemann, 2008): a fundamental motive, making agents strive to predict and fit some exogenous fundamental value, a coordination motive, giving them an incentive to match the conventional value

\footnotetext{
*We are grateful to Rabah Amir for useful discussions. We also want to thank Pedro Gardete, two anonymous referees, and the participants to the workshop "Coordination in Economics" organized from May $29^{\text {th }}$ to June $3^{\text {rd }} 2017$ at the Fondation des Treilles, plus the Foundation itself for the corresponding financial support.

$\dagger$ Univ Lyon, CNRS, GATE UMR 5824, F-69130 Ecully, France; email: cornand@gate.cnrs.fr.

${ }^{\ddagger}$ BETA, University of Strasbourg, 61 avenue de la Forêt Noire, 67085 Strasbourg Cedex, France; email: rdsf@unistra.fr; and Católica Lisbon School of Business and Economics.
} 
to be set by the market, and a competition motive, making them better off when beating the market.

Should information be perfect, the fundamental and the coordination motives would be compatible: all agents would simply coordinate on the fundamental value. As information becomes imperfect, blurring the fundamental, and dispersed, obstructing coordination, a conflict emerges between the fundamental and coordination motives. The competition motive introduces a further conflict, even in the absence of informational frictions, since pursuing the coordination and the competition motives means meeting and beating the market at the same time. While the conflict between the fundamental and the coordination motives has been largely explored in the beauty contest literature, less attention has been paid to the influence of the competition motive, which we want to emphasize now as a source of inefficiency under perfect as well as under imperfect and dispersed information.

Our contribution consists in building a unified framework bridging the gap between team and competition issues. On the one hand, this unified framework allows us to reconsider the social value of private and public information in a large category of contexts going beyond the Keynesian beauty contest, since they extend from situations of strategic complementarity generated by the coordination motive to situations of strategic substitutability resulting from an anti-coordination motive, when agents want to move away from each other. On the other hand, it enables us to formulate direct comparisons between the performances of price and quantity games when information is imperfect and dispersed.

To obtain the unified framework and capture the specific impact of the competition motive, we model an economy with a simple multidivisional company, ${ }^{1}$ where each division supplies a diversified good and is assigned to maximize a convex combination of its own profit and of the company joint profit. We thus obtain as polar cases full competition (leading to a standard oligopolistic equilibrium) and full cooperation (entailing the collusive solution). ${ }^{2}$ The latter outcome, the solution to a team problem, is of course efficient from the company's point of view and may be taken as a benchmark.

The model is studied in the context of both price and quantity games. Introducing the competition motive has two effects. First, independently from informational issues, this motive distorts the equilibrium strategies away from their

\footnotetext{
${ }^{1}$ On the multidivisional form of organization, see Mahoney (1992).

${ }^{2}$ As suggested by the very terminology we are using, an alternative interpretation of our model is that of an oligopolistic market for diversified goods, in which firms engage in a more or less cooperative conduct. Related literature has generally considered only the two extreme conducts, resulting in standard Cournot and Bertrand oligopoly equilibria or in the monopoly solution (Amir et al., 2010, and Amir et al., 2016, respectively, are good representatives of this methodological choice). We consider instead any possible semi-cooperative conduct, but the conditions underlying the choice and the enforcement of such conduct by an independent firm are not contemplated in the present analysis. Using a model of differentiated duopoly, Cornand and Dos Santos Ferreira (2017) focus upon the strategic choice by firm owners of the degree of cooperation ruling the conduct of firm managers, hence upon the endogeneity of the relative weight to be attributed to the competition (and coordination) motive(s).
} 
efficient value, thus generating a (price or quantity) strategy distortion. Second, under coexisting public and private information on the stochastic fundamental (the market size), the competition motive distorts the relative weights put on the two kinds of information, generating an informational distortion, which countervails the effect of the coordination (or anti-coordination) motive. In both price and quantity games, the informational distortion generated by the competition motive thus translates into an inefficiently low weight on the public signal in the presence of the coordination motive (hence under strategic complementarity), and into an inefficiently high weight on the public signal in the presence of the anti-coordination motive (hence under strategic substitutability).

After examining the well-known case of perfect information where the competition motive is responsible for the sole strategy distortion, we first establish some results regarding the social value of information. In the absence of the competition motive, but also when this motive combines with the anti-coordination motive (hence under strategic substitutability), the social value of both public and private information is always positive. By contrast, when it combines with the coordination motive (hence under strategic complementarity), while the social value of public information is still positive, that of private information becomes negative for a high enough intensity of competition. Indeed, increasing the precision of either public or private information directly helps divisions better predict the fundamentals, but may also augment the informational distortion. This additional effect of an increase in the precision of private information results under strategic complementarity in an excessive weight put on the private signal. The negative indirect effect eventually dominates the positive direct effect as the intensity of competition becomes larger and larger, reducing the importance of the fundamental motive.

We finally compare from a welfare point of view the outcomes of the price and quantity games. Under imperfect and dispersed information, the efficient and socially optimal ${ }^{3}$ uses of information coincide in the absence of the competition motive, ensuring higher performance levels under the influence of anticoordination rather than coordination (hence under strategic substitutability rather than strategic complementarity). This result obtains under the sole influence of the coordination/anti-coordination motive and only for informational reasons. However, the competition motive may reverse the result, as it generates strategy and informational distortions with opposite effects on social welfare. If the information quality is low (under small precisions of both public and private signals), the effect of the informational distortion dominates the effect of the strategy distortion, so that profit and social welfare are higher with the coordination motive (hence under strategic complementarity). Interestingly, the informational distortion generated by the competition motive thus mitigates the perfect information result in favor of anti-coordination (hence of strategic

\footnotetext{
${ }^{3}$ Our economy is reduced to a representative consumer with quasilinear utility and to a multidivisional company. This modelling approach rationalizes the standard view of "social" welfare (here identified with consumer's added utility) as the sum of the consumer surplus and distributed profits. Clearly, in a more diversified economy with profits distributed to only part of the consumers, the evaluation of social welfare would be more problematic.
} 
substitutability).

The paper contributes to two main strands of the literature. First, it belongs to the growing literature on the social value of information initiated by Morris and Shin (2002), highlighting the conflict between the fundamental and the coordination motives, and showing that an increase in the precision of public information is harmful in a context of strategic complementarity if private information is already precise enough. This result is largely due to the presence of the sole fundamental motive in the social welfare function, the coordination motive being exactly counterbalanced by the competition motive in Morris and Shin's framework. Angeletos and Pavan (2007) show that Morris and Shin's result must indeed be qualified, in particular by referring to the relation between the equilibrium and the efficient uses of information. Also, Ui and Yoshizawa (2015) characterize the social value of information in symmetric quadratic-payoff games for a larger class of environments than Angeletos and Pavan. ${ }^{4}$ Both propose IO (Bertrand and Cournot) applications, without pointing to the source of the discrepancy between the equilibrium and efficient uses of information, namely the competition motive. ${ }^{5}$ The same IO illustrations of beauty contest games, used to emphasize the conflict between the fundamental and the coordination motives, appear in Myatt and Wallace (2012, 2015, 2018). By taking as the fundamental targets pursued by the oligopolistic firms the Bertrand and the Cournot equilibrium strategies, Myatt and Wallace merge the fundamental and competition motives. Such a procedure does not allow to consider the effect of the competition motive separately, which is our aim in the present paper. We take instead the collusive strategies as the fundamental targets, which allows us to disentangle the fundamental and the competition motives.

Second, the paper extends to a context of imperfect and dispersed information the systematic comparison of price and quantity competition initiated by Singh and Vives (1984) for a differentiated duopoly with linear demand (recent contributions, also under perfect information, are provided by Amir and Jin, 2001, and Amir, Erickson and Jin, 2017). In the same vein, we observe the development of a literature on the aggregation of private information in oligopolies with either demand or cost uncertainty ${ }^{6}$ (Raith, 1996, Vives, 1988, 2011). This literature goes beyond the scope of the present paper in dealing with firms' strategic decisions concerning information sharing and acquisition, or else with private information revelation at equilibrium. Nonetheless, our work could have

\footnotetext{
${ }^{4}$ In particular, they also establish results for certain games with a finite number of players, which are useful for applications such as oligopoly games. Their main contribution is to provide a necessary and sufficient condition for welfare to increase with either the precision of public or the precision of private information, starting from an arbitrary precision of these sources. Welfare is represented as a linear combination of the volatility of the average action and the dispersion of the individual actions.

${ }^{5} \mathrm{Ui}(2009)$ has already built a bridge between the beauty contest literature and the team literature (which goes back to Radner, 1962), focusing on a team problem similar to our collusion benchmark. His result generalizes Cremer (1990), emphasizing that "shared knowledge is better than diversified knowledge if and only if a team exhibits strategic complementarity" (Ui, 2009, p. 273).

${ }^{6}$ Our framework addresses only the first type of uncertainty.
} 
some implications of interest in terms of information sharing about uncertain demand. We show indeed that information sharing - viewed, like in Angeletos and Pavan (2007), as an increase in the precision of public information is profit enhancing under both Bertrand and Cournot regimes of competition. While this result is in line with Amir et al. (2010, Corollary 2) and finds some empirical support in the recent though scare empirical literature (see notably Gardete, 2016), it stands in contrast to that of most of the theoretical literature (as developped by Gal-Or, 1985, Novshek and Sonnenschein, 1982, Li, 1985, Sakai 1985). As emphasized by Raith (1996, p. 263) in his synthetic model, a general result in this literature is that "with a common value and strategic complements, complete information pooling is an equilibrium of the two-stage game (which is efficient from the viewpoint of the firms), regardless of all other parameters. With a common value and strategic substitutes, no pooling is the equilibrium solution. This solution is efficient in Cournot markets with homogeneous goods and inefficient for a large degree of product differentiation." The present article differs from this literature in several respects. First, it proposes a more general profit function by possibly including the total profit in the objective function of each division. Second, while this literature generally considers only private signals (and their potential revelation as multiple public signals), the information structure of the present paper considers the simultaneous provision of public and private information, which allows analyzing how firms use public relatively to private information. Moreover, this literature usually considers prior information on demand conditions, while we do not allow for prior information.

The remaining of the paper is structured as follows. Section 2 presents the set-up of the economy. Section 3 focuses on the perfect information benchmark, emphasizing the strategy distortion generated by the competition motive, while section 4 examines equilibrium and welfare under dispersed information, emphasizing the informational distortion. Section 5 concludes.

\section{The economy}

We consider a simple economic sector composed of a representative consumer and a multidivisional company.

\subsection{The representative consumer}

We assume that the representative consumer's utility is symmetric ${ }^{7}$ quadratic with respect to the differentiated goods supplied by the company divisions and quasi-linear with respect to a composite good representing the rest of the economy.

\footnotetext{
${ }^{7}$ Symmetry is a convenient but restrictive assumption. Amir and Jin (2001) and Amir et al. (2010) compare Cournot and Bertrand equilibria, also with linear demand, but without imposing symmetry. Asymmetry allows in particular taking firm-specific demand shocks into account.
} 
The representative consumer, endowed with positive wealth $w$, buys at prices $\mathbf{p} \in \mathbb{R}_{++}^{n}$ a basket $\mathbf{q} \in \mathbb{R}_{+}^{n}$ of $n$ differentiated goods to the $n$ divisions of the company, plus a quantity $z \in \mathbb{R}_{+}$of a composite numeraire good to a competitive industry, so as to maximize, under the budget constraint pq $+z \leq w$ utility

$$
U(\mathbf{q}, z)=u(\mathbf{q})+z
$$

with

$$
u(\mathbf{q})=2 \theta \sum_{i=1}^{n} q_{i}-\frac{1}{2} \sum_{i=1}^{n} q_{i}^{2}-\frac{\gamma}{2}\left(\sum_{i=1}^{n} q_{i}\right)^{2} .
$$

The positive parameter $\theta$ is an index of market size and $|\gamma|$ is the degree of interdependence between goods, goods being substitutes if $\gamma>0$, complements if $-1 / n<\gamma<0$, independent if $\gamma=0$.

The first order condition for utility maximization leads directly to the inverse demand function for each good $i=1, \ldots, n$ :

$$
p_{i}=2 \theta-q_{i}-n \gamma \underbrace{\frac{1}{n} \sum_{j=1}^{n} q_{j}}_{q} \equiv P_{i}(\mathbf{q}) .
$$

From this equation system we easily obtain the demand function for each good $i=1, \ldots, n$ :

$$
q_{i}=\frac{1}{1+n \gamma}(2 \theta-(1+n \gamma) p_{i}+n \gamma \underbrace{\frac{1}{n} \sum_{j=1}^{n} p_{j}}_{p}) \equiv Q_{i}(\mathbf{p}) .
$$

\subsection{The multidivisional company}

The company assigns as objective to each division $i=1, \ldots, n$ a convex combination of the division's own profit and of the joint profit of all divisions. The relative weight $\lambda$ put on the former is a measure of the strength of the competition motive within the organization. We thus obtain as polar cases: the fully non-cooperative conduct (oligopolistic competition between the divisions) for $\lambda=1$, and the fully cooperative conduct (collusion between the divisions) for $\lambda=0$. More explicitly, the program of each division $i$ is, assuming zero production costs,

$$
\max _{p_{i}}\left[\lambda p_{i} Q_{i}(\mathbf{p})+(1-\lambda) \mathbf{p Q}(\mathbf{p})\right]
$$

where $\boldsymbol{Q}(\mathbf{p}) \equiv\left(Q_{1}(\mathbf{p}), \ldots, Q_{n}(\mathbf{p})\right)$, when divisions play in prices, and

$$
\max _{q_{i}}\left[\lambda q_{i} P_{i}(\mathbf{q})+(1-\lambda) \mathbf{q} \mathbf{P}(\mathbf{q})\right],
$$

where $\mathbf{P}(\mathbf{q}) \equiv\left(P_{1}(\mathbf{q}), \ldots, P_{n}(\mathbf{q})\right)$, when they play in quantities. 


\section{The perfect information benchmark: the com- petition motive and the strategy distortion}

We first refer to the benchmark of perfect information, considering successively equilibrium and welfare in the price and quantity games, before directly comparing the two kinds of games.

\subsection{Equilibrium}

- The price game

Instead of referring to the program (1), we can alternatively refer to an equivalent program consisting in minimizing the loss of the objective assigned to division $i$ relative to the collusive profit (obtained for $\lambda=0$ with all prices equal to the fundamental $\theta$ ):

$$
\theta Q_{i}(\theta, \ldots, \theta)-\left[\lambda p_{i} Q_{i}(\mathbf{p})+(1-\lambda) \mathbf{p Q}(\mathbf{p})\right]=\frac{1}{1+n \gamma} L^{P}\left(p_{i}, p_{-i}, \theta\right)
$$

with $p_{-i}=\left(\sum_{j \neq i} p_{j}\right) /(n-1)$ and the loss function $L^{P}$ (to be minimized in $\left.p_{i}\right)$

$$
\begin{aligned}
& L^{P}\left(p_{i}, p_{-i}, \theta\right) \\
& =\underbrace{\left(p_{i}-\theta\right)^{2}}_{\text {fundamental motive }}+(n-1) \gamma(\underbrace{\left(p_{i}-p_{-i}\right)^{2}}_{\text {(anti-)coordination motive }}+\lambda \underbrace{p_{-i}\left(p_{i}-p_{-i}\right)}_{\text {competition motive }}) \\
& +(n-1)(1-\lambda) \underbrace{\left(\frac{1+n \gamma}{n-1} \sum_{j \neq i} p_{j}^{2}-n \gamma p_{-i}^{2}-2 \theta p_{-i}\right)}_{\text {externality }} .
\end{aligned}
$$

This loss function is obviously reminiscent of the loss function introduced by Morris and Shin (2002) in their seminal modelling of the beauty contest, with its three motives: ${ }^{8}$ the fundamental, the coordination and the competition

\footnotetext{
${ }^{8}$ Morris and Shin (2002) loss function is indeed the sum of a fundamental, a coordination and a competition motives:

$$
(1-r)\left(a_{i}-\theta\right)^{2}+r\left(a_{i}-\bar{a}\right)^{2}-r \frac{1}{n} \sum_{j}\left(a_{j}-\bar{a}\right)^{2},
$$

with given weights $1-r, r$ and $-r$, respectively, and involving individual and mean strategies $a_{i}$ and $\bar{a}$. An important difference with respect to our own loss function is that, by assuming a continuum of agents, Morris and Shin make the competition motive inoperative regarding individual decisions. Another important difference concerns aggregate losses: the competition motive exactly balances the coordination motive in the Morris and Shin specification, so that the fundamental motive stands alone as a component of social welfare. By contrast, the competition motive vanishes under symmetry by aggregation of our loss functions, so that coordination contributes in our case to agents' welfare.
} 
motives. ${ }^{9}$ We formulate three remarks concerning the present loss function.

First, the fundamental motive stands naturally alone when there is a single division $(n=1)$ or when the goods are independent $(\gamma=0)$. Correspondingly, its relative weight diminishes as the level $(n-1)|\gamma|$ of interdependence between divisions increases (either through the number $n-1$ of competitors of each division or through the degree $|\gamma|$ of interdependence between goods).

Second, the coordination motive becomes in fact an anti-coordination motive when we switch from the case of substitutable goods $(\gamma>0)$ to the case of complementary goods $(\gamma<0)$. There is however more than a simple change of signs. An important difference between the two cases is that, as the level of interdependence between divisions tends to its maximum, the fundamental motive eventually vanishes relative to the coordination motive (since $(n-1)|\gamma| \rightarrow \infty$ as $\gamma \rightarrow \infty$ ), whereas it always dominates the anti-coordination motive (since $(n-1)|\gamma| \rightarrow 1-1 / n<1$ as $\gamma \rightarrow-1 / n)$.

Third, symmetry in strategy deviations from the targets is broken by the competition motive, which introduces a downward (upward) bias when the coordination (anti-coordination) motive prevails. The weight on the competition motive is the intensity of competition, obtained by multiplying the level $(n-1)|\gamma|$ of interdependence between divisions and the strength $\lambda$ of the competition motive, thus combining two structural characteristics and a conduct characteristic of competition.

The first order condition for minimization of $L^{P}\left(\cdot, p_{-i}, \theta\right)$ gives the best reply function

$$
p_{i}=\frac{\theta+(n-1) \gamma(1-\lambda / 2) p_{-i}}{1+(n-1) \gamma} .
$$

Naturally, the coordination motive (for $\gamma>0$ ) implies strategic complementarity, and the anti-coordination motive (for $\gamma<0$ ) strategic substitutability.

By symmetry $\left(p_{i}=p_{-i}\right)$, we deduce from the best reply function the equilibrium price $p^{P}$ (and the corresponding quantity $q^{P}$ ), equal for all $i$ and depending upon the strength $\lambda$ of the competition motive:

$$
p^{P}(\lambda)=\underbrace{\frac{1}{1+(n-1) \gamma \lambda / 2}}_{K^{P}(\lambda)} \theta, q^{P}(\lambda)=\underbrace{\frac{1+(n-1) \gamma \lambda}{1+(n-1) \gamma \lambda / 2}}_{2-K^{P}(\lambda)} \frac{\theta}{1+n \gamma} .
$$

The equilibrium price $p^{P}$, equal to the fundamental in the absence of the competition motive, is otherwise distorted, taking lower (higher) values in the presence of the coordination (anti-coordination) motive, in other words under strategic

\footnotetext{
${ }^{9}$ Myatt and Wallace (2012) assume a continuum of competitors supplying differentiated substitutes, and rewrite directly the profit function as a loss function (up to its sign):

$p_{i}\left((2-\beta) \theta-p_{i}+\beta p\right)=-(1-\beta / 2)\left(p_{i}-\theta\right)^{2}-(\beta / 2)\left(p_{i}-p\right)^{2}+(1-\beta / 2) \theta^{2}+(\beta / 2) p^{2}$,

with $\beta \in(0,1)$. This loss function exhibits the sole fundamental and coordination motives, but the competition motive is actually merged with the fundamental motive, since the fundamental $\theta$ is the (monopolistically) competitive price, not the collusive price $\theta(1-\beta / 2) /(1-\beta)$ as in our framework.
} 
complementarity (substitutability). Of course, we obtain an opposite effect on the equilibrium quantity $q^{P}$. The distortion - a consequence of the bias introduced by the competition motive - is the larger the stronger the intensity of competition $(n-1)|\gamma| \lambda$.

- The quantity game

Proceeding in the same way, we consider the loss of the objective assigned to division $i$ relative to the collusive profit (obtained for $\lambda=0$ with all quantities equal to $\theta /(1+n \gamma))$ as

$$
\begin{aligned}
& \frac{\theta}{1+n \gamma} P_{i}\left(\frac{\theta}{1+n \gamma}, \ldots, \frac{\theta}{1+n \gamma}\right)-\left[\lambda q_{i} P_{i}(\mathbf{q})+(1-\lambda) \mathbf{q} \mathbf{P}(\mathbf{q})\right] \\
= & (1+n \gamma) L^{Q}\left(q_{i}, q_{-i}, \theta\right)
\end{aligned}
$$

with $q_{-i}=\left(\sum_{j \neq i} q_{j}\right) /(n-1)$ and the loss function $L^{Q}$ (to be minimized in $\left.q_{i}\right)$

$$
\begin{aligned}
& L^{Q}\left(q_{i}, q_{-i}, \theta\right) \\
= & \underbrace{\left(q_{i}-\frac{\theta}{1+n \gamma}\right)^{2}}_{\text {fundamental motive }}-\frac{(n-1) \gamma}{1+n \gamma}(\underbrace{\left(q_{i}-q_{-i}\right)^{2}}_{\text {(anti-)coordination motive }}+\lambda \underbrace{q_{-i}\left(q_{i}-q_{-i}\right)}_{\text {competition motive }}) \\
& +\frac{(n-1)(1-\lambda)}{1+n \gamma} \underbrace{\left(\frac{1}{n-1} \sum_{j \neq i} q_{j}^{2}+n \gamma q_{-i}^{2}-2 \theta q_{-i}\right)}_{\text {externality }} .
\end{aligned}
$$

Again, this loss function is reminiscent of the one in the beauty contest model of Morris and Shin (2002). The observations formulated for $L^{P}$ stand for $L^{Q}$, but the sign of the weight put on the coordination and competition motives is naturally reversed, an anti-coordination motive prevailing now when goods are substitutes. As in the price game, when the intensity of competition tends to its maximum, this weight tends to infinity if applied to the coordination motive but it remains dominated if applied to the anti-coordination motive.

The first order condition for minimizing $L^{Q}\left(\cdot, q_{-i}, \theta\right)$ gives the best reply function

$$
q_{i}=\frac{\theta-(n-1) \gamma(1-\lambda / 2) q_{-i}}{1+\gamma}
$$

As in the case of the price game, coordination generates strategic complementarity, anti-coordination strategic substitutability. By symmetry $\left(q_{i}=q_{-i}\right)$, we deduce from the best reply function the equilibrium quantity $q^{Q}$ (and the corresponding price $p^{Q}$ ), the same for each $i$ and depending upon the strength of the competition motive $\lambda$ :

$$
q^{Q}(\lambda)=\underbrace{\frac{1}{1+n \gamma-(n-1) \gamma \lambda / 2}}_{K^{Q}(\lambda)} \theta, p^{Q}(\lambda)=\underbrace{\frac{1+n \gamma-(n-1) \gamma \lambda}{1+n \gamma-(n-1) \gamma \lambda / 2}}_{2-(1+n \gamma) K^{Q}(\lambda)} \theta .
$$


Observe that under full cooperation of the company divisions, $p^{Q}(0)=$ $p^{P}(0)=\theta$ and $q^{Q}(0)=q^{P}(0)=\theta /(1+n \gamma)$. Under perfect information and a vanishing competition motive, it is indifferent to play in prices or in quantities. However, if $\lambda>0$, the equilibrium price is distorted in the same way as in the price game. Notice further that $p^{Q}(\lambda)>p^{P}(\lambda)$ independently of the nature of the interdependence between goods: the strategy distortion is just larger under strategic complementarity, pushing the price $p^{P}$ downwards when $\gamma>0$, and the price $p^{Q}$ upwards when $\gamma<0$.

The following proposition summarizes the consequences of introducing the competition motive in the two games.

Proposition 1 The competition motive generates a strategy distortion in both price and quantity games: as it becomes stronger (through a larger $\lambda$ ), equilibrium prices become lower (higher) and equilibrium quantities higher (lower) when goods are substitutes (complements). This distortion increases with the intensity of competition $(n-1)|\gamma| \lambda$, and is larger when the coordination motive prevails, hence under strategic complementarity (in the price game when goods are substitutes, and in the quantity game when they are complements).

\subsection{Welfare}

Because of the representative consumer assumption, it is straightforward to proceed to welfare analysis. Consumer's utility at equilibrium quantities (be it $\mathbf{q}^{*}=\mathbf{q}^{P}(\lambda)$ or $\left.\mathbf{q}^{*}=\mathbf{q}^{Q}(\lambda)\right)$ can be decomposed as follows:

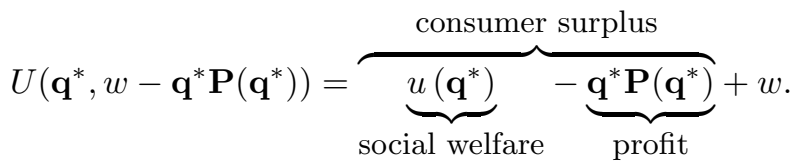

- The price game

We compute profit, social welfare and the consumer surplus successively. By symmetry and using (5), the company profit

$$
\Pi^{P}(\lambda)=n p^{P}(\lambda) q^{P}(\lambda)=\frac{n \theta^{2}}{1+n \gamma}\left(2-K^{P}(\lambda)\right) K^{P}(\lambda)
$$

is seen to be equal to the collusive profit $n \theta^{2} /(1+n \gamma)$ multiplied by the factor $1-\left(1-K^{P}(\lambda)\right)^{2}$, which is always smaller than one if $\lambda>0$, and decreasing in the intensity of competition $(n-1)|\gamma| \lambda$. As expected, the company profit is maximized at $\lambda=0$. Social welfare

$$
u^{P}(\lambda)=\frac{n \theta^{2}}{1+n \gamma}\left(2-K^{P}(\lambda)\right)\left(1+K^{P}(\lambda) / 2\right)=\frac{n \theta^{2}}{1+n \gamma}\left[2-\left(K^{P}(\lambda)\right)^{2} / 2\right]
$$

and the consumer surplus

$$
u^{P}(\lambda)-\Pi^{P}(\lambda)=\frac{n \theta^{2}}{1+n \gamma} 2\left[1-K^{P}(\lambda) / 2\right]^{2}
$$


are by contrast increasing (decreasing) in the intensity of competition if goods are substitutes (complements). Hence, they are both maximized at $\lambda=1$ when goods are substitutes, and at $\lambda=0$ when they are complements. As already shown by Cournot (1838, ch. IX), producers' and consumers' interests converge when goods are complements, whereas they diverge when goods are substitutes.

- The quantity game

By symmetry and using (8), the company profit

$$
\Pi^{Q}(\lambda)=n p^{Q}(\lambda) q^{Q}(\lambda)=\frac{n \theta^{2}}{1+n \gamma}\left[1-\left((1+n \gamma) K^{Q}(\lambda)-1\right)^{2}\right]
$$

is again seen to be equal to the collusive profit multiplied by a factor (between brackets) which is always smaller than one if $\lambda>0$, and which is decreasing in the intensity of competition $(n-1)|\gamma| \lambda$, so that profit is maximized at $\lambda=0$. Social welfare

$$
u^{Q}(\lambda)=\frac{n \theta^{2}}{1+n \gamma} 2\left[1-\left(\frac{(1+n \gamma) K^{Q}(\lambda)}{2}-1\right)^{2}\right]
$$

and the consumer surplus

$$
u^{Q}(\lambda)-\Pi^{Q}(\lambda)=\frac{n \theta^{2}}{1+n \gamma} \frac{(1+n \gamma)^{2}\left(K^{Q}(\lambda)\right)^{2}}{2}
$$

are, as in the price game, both increasing (decreasing) in the intensity of competition if goods are substitutes (complements). Hence, social welfare and consumer surplus are again maximized at $\lambda=1$ when goods are substitutes, and at $\lambda=0$ when they are complements.

We recall in the following proposition the preceding observations.

Proposition 2 Under perfect information, profit is decreasing in the intensity of competition $(n-1)|\gamma| \lambda$ (as the strategy distortion becomes more severe) in both price and quantity games. Social welfare and the consumer surplus are increasing (decreasing) in the intensity of competition if goods are substitutes (complements): the interests of the company and of the consumer diverge when goods are substitutes, but converge when they are complements.

\subsection{Price vs. quantity games}

In this subsection we compare price and quantity games from a welfare perspective. Take first, using equations (10) and (13), the difference in profits obtained when playing each kind of strategies.

$$
\Pi^{Q}(\lambda)-\Pi^{P}(\lambda)=\frac{n \theta^{2}}{1+n \gamma}\left(\left(1-K^{P}(\lambda)\right)^{2}-\left((1+n \gamma) K^{Q}(\lambda)-1\right)^{2}\right)
$$


There are two cases in which this expression vanishes. The first case is when $\gamma=0$, entailing $K^{P}(\lambda)=(1+n \gamma) K^{Q}(\lambda)=1$ for any $\lambda$ : it is indifferent to play in quantities or in prices when goods are independent. The second is when $\lambda=0$, since $K^{P}(0)=(1+n \gamma) K^{Q}(0)=1$, equilibrium prices being in both games equal to $\theta$, as we have already pointed out. Thus, when the competition motive is suppressed, it is indifferent for the company to play in quantities or in prices.

Otherwise, if $\lambda>0$, it is easy to check from (5) and (8), that $K^{P}(\lambda)<$ $1<(1+n \gamma) K^{Q}(\lambda)$ for $\gamma>0,(1+n \gamma) K^{Q}(\lambda)<1<K^{P}(\lambda)$ for $\gamma<0$, and $K^{P}(\lambda)+(1+n \gamma) K^{Q}(\lambda)<2$ in both cases. Hence, $\Pi^{Q}(\lambda)-\Pi^{P}(\lambda)$ has the sign of $\gamma$ : profits will always be larger when playing in substitutable strategies, that is, under the anti-coordination motive.

As to social welfare and the consumer surplus, using equations (11), (12), (14) and (15), we have

$$
u^{Q}(\lambda)-u^{P}(\lambda)=\frac{n \theta^{2}}{1+n \gamma} \frac{\left(K^{P}(\lambda)\right)^{2}-\left(2-(1+n \gamma) K^{Q}(\lambda)\right)^{2}}{2}<0,
$$

and

$$
\begin{aligned}
& \left(u^{Q}(\lambda)-\Pi^{Q}(\lambda)\right)-\left(u^{P}(\lambda)-\Pi^{P}(\lambda)\right) \\
= & \frac{n \theta^{2}}{1+n \gamma}\left(\frac{\left((1+n \gamma) K^{Q}(\lambda)\right)^{2}-\left(2-K^{P}(\lambda)\right)^{2}}{2}\right)<0 .
\end{aligned}
$$

The price game is always preferable from the point of view of the consumer and always socially optimal. Convergence of interests between the company and the consumer when goods are complements and divergence when they are substitutes are again apparent.

To recall,

Proposition 3 For the company as well as for the consumer, it is indifferent to play in prices or in quantities when the goods are independent $(\gamma=0)$ or else in the absence of the competition motive $(\lambda=0)$. Otherwise, the consumer always prefers the price game, which is socially optimal. For the company, however, playing under the anti-coordination motive (and thus minimizing the strategy distortion) is preferable, so that the price game leads to a higher profit only when goods are complements.

\section{Dispersed information: the competition mo- tive and the informational distortion}

We shall now assume that the company divisions do not know the realization $\theta$ of the fundamental, on which they only receive two noisy signals, ${ }^{10}$ a public

\footnotetext{
${ }^{10}$ As in Myatt and Wallace (2012), players are assumed to share an improper prior over $\theta$, which simplifies computations. We analyze the consequence of such an assumption in the
} 
signal $y=\theta+\eta$, with $\eta \sim N(0,1 / \alpha)$ and a private signal $x_{i}=\theta+\varepsilon_{i}$, with $\varepsilon_{i} \sim N(0,1 / \beta)$. As in Morris and Shin (2002), the random variables $\eta$ and $\varepsilon_{1}, \ldots, \varepsilon_{n}$ are independent. ${ }^{11}$ We assume that information is imperfect $(\alpha<\infty$ and $\beta<\infty)$, and also that it is dispersed $(\beta>0)$.

\subsection{Equilibrium}

Division $i$ forms expectations on the realization $\theta$ of the fundamental, but also on the mean strategy $p_{-i}$ or $q_{-i}$ of the other divisions, according to the game type, by referring to the two signals $y$ and $x_{i}$. We assume that it responds linearly to those two signals, targeting the expected best price or quantity reply to others' expected strategies (conditional on the two signals): $p_{i}=\zeta_{i}^{P} y+\xi_{i}^{P} x_{i}$ or $q_{i}=\zeta_{i}^{Q} y+\xi_{i}^{Q} x_{i}$. This amounts for division $i$ to choose in fact a non-negative strategy pair $\left(\zeta_{i}^{P}, \xi_{i}^{P}\right)$ or $\left(\zeta_{i}^{Q}, \xi_{i}^{Q}\right)$.

- The price game

The choice of $\left(\zeta_{i}^{P}, \xi_{i}^{P}\right)$ targets the expected best price reply, which we can infer from equation (4):

$$
\begin{aligned}
\zeta_{i}^{P} y+\xi_{i}^{P} x_{i} & =\mathbb{E}\left(\frac{\theta+\gamma(1-\lambda / 2) \sum_{j \neq i}\left(\zeta_{j}^{P} y+\xi_{j}^{P} x_{j}\right)}{1+(n-1) \gamma} \mid y, x_{i}\right) \\
& =\frac{\mathbb{E}\left(\theta \mid y, x_{i}\right)\left(1+\gamma(1-\lambda / 2) \sum_{j \neq i} \xi_{j}^{P}\right)+\gamma(1-\lambda / 2) y \sum_{j \neq i} \zeta_{j}^{P}}{1+(n-1) \gamma} .
\end{aligned}
$$

As $\mathbb{E}\left(\theta \mid y, x_{i}\right)=\left(\alpha y+\beta x_{i}\right) /(\alpha+\beta)$,

$$
\xi_{i}^{P}=\frac{\beta}{\alpha+\beta} \frac{1+\gamma(1-\lambda / 2) \sum_{j \neq i} \xi_{j}^{P}}{1+(n-1) \gamma},
$$

implying at equilibrium

$$
\xi_{i}^{P}=\xi^{P}=\frac{1}{1+\alpha / \beta+(n-1) \gamma(\lambda / 2+\alpha / \beta)} .
$$

Appendix, by comparing our results on the welfare effects of changes in the precision of public and private information with those of Angeletos and Pavan (2007), who do not make the same assumption. Also notice that we assume the same fundamental random variable $\theta$, perturbing the consumer's utility function, independently of the competition regime. We thus take the same approach as Amir et al. (2016), in contrast to most of the literature (including Angeletos and Pavan, 2007), which approaches demand shocks under Bertrand competition through a random intercept of the direct demand function - a derived variable.

${ }^{11}$ Assuming, as most of the literature, normality of the noise distributions may lead to states where demand is negative. We are implicitly assuming that the signal precisions are high enough for the probability of such an event to be small (see Vives, 1984, p. 77). Amir et al. (2010) free themselves from specific assumptions on the distributions of shocks and signals, but they address cost, not demand, uncertainty in a model of firm-specific information sharing. 
The next step allows us to determine

$$
\zeta_{i}^{P}=\zeta^{P}=\frac{1}{1+\alpha / \beta+(n-1) \gamma(\lambda / 2+\alpha / \beta)} \frac{1+(n-1) \gamma}{1+(n-1) \gamma(\lambda / 2)} \frac{\alpha}{\beta} .
$$

The strategy sum

$$
\zeta^{P}+\xi^{P}=\frac{1}{1+(n-1) \gamma \lambda / 2}=K^{P}(\lambda)
$$

does not depend upon the quality of information, as given by the precisions $\alpha$ and $\beta$. It is equal to the coefficient multiplying the fundamental to obtain the equilibrium price under perfect information. The strategy distortion with its effects derived in the benchmark case is still at work, implying in particular that "the Bertrand price reacts too little to $\theta$ as compared to the monopoly price" (Angeletos and Pavan, 2007, p. 1129), meaning in our framework that $K^{P}(1)<K^{P}(0)$, a fact which depends upon the strategy distortion and which is not modified by the imperfection and dispersion of the information.

The novelty introduced by assuming imperfect and dispersed information comes from the relative weights $\zeta /(\xi+\zeta)$ and $\xi /(\xi+\zeta)$ put, respectively, on the public and private signals, with

$$
\frac{\zeta^{P}}{\xi^{P}+\zeta^{P}}=\frac{(1+(n-1) \gamma) \alpha / \beta}{(1+(n-1) \gamma) \alpha / \beta+1+(n-1) \gamma \lambda / 2} \equiv \kappa^{P}(\lambda)
$$

and $\xi^{P} /\left(\xi^{P}+\zeta^{P}\right)=1-\kappa^{P}(\lambda)$. The efficient (relative) weight on the public signal is obviously $\kappa^{P}(0)$, which takes into account the fundamental and the coordination (or anti-coordination) motives, without the competition motive. The fundamental motive alone would lead to a relative weight on the public signal given by its relative precision $\alpha /(\alpha+\beta)$. However, for the sake of coordination, and as long as the goods are substitutes $(\gamma>0)$, hence prices strategic complements, the efficient (relative) weight on the public signal

$$
\kappa^{P}(0)=\frac{(1+(n-1) \gamma) \alpha / \beta}{(1+(n-1) \gamma) \alpha / \beta+1}
$$

is increasing in the level of interdependence between divisions, as measured by $(n-1)|\gamma|$. Obviously, it is decreasing in the level of interdependence between divisions under the anti-coordination motive $(\gamma<0)$, hence under strategic substitutability.

Introducing the competition motive leads to an informational distortion, by decreasing (increasing) $\kappa^{P}(\lambda)$ under the coordination (anti-coordination) motive, thus by countervailing the effect of this motive. In relative terms, this informational distortion is

$$
\frac{\left|\kappa^{P}(0)-\kappa^{P}(\lambda)\right|}{\kappa^{P}(0)}=\frac{(n-1)|\gamma| \lambda / 2}{(1+(n-1) \gamma) \alpha / \beta+1+(n-1) \gamma \lambda / 2},
$$

increasing in the intensity of competition $(n-1)|\gamma| \lambda$ and decreasing in the ratio of precisions $\alpha / \beta$. 
- The quantity game

The choice of the strategy pair $\left(\zeta_{i}^{Q}, \xi_{i}^{Q}\right)$ now targets the expected best quantity reply, which we can infer from equation (7):

$\zeta^{Q} y+\xi^{Q} x_{i}=\frac{\mathbb{E}\left(\theta \mid\left(y, x_{i}\right)\right)\left(1-(n-1) \gamma(1-\lambda / 2) \xi^{Q}\right)-(n-1) \gamma(1-\lambda / 2) \zeta^{Q} y}{1+\gamma}$

so that

$$
\begin{aligned}
\xi^{Q} & =\frac{1}{1+n \gamma-(n-1) \gamma \lambda / 2+(1+\gamma) \alpha / \beta}, \text { and } \\
\zeta^{Q} & =\frac{1}{1+n \gamma-(n-1) \gamma \lambda / 2+(1+\gamma) \alpha / \beta} \frac{(1+\gamma) \alpha / \beta}{1+n \gamma-(n-1) \gamma \lambda / 2}
\end{aligned}
$$

with a strategy sum

$$
\zeta^{Q}+\xi^{Q}=\frac{1}{1+n \gamma-(n-1) \gamma \lambda / 2}=K^{Q}(\lambda) .
$$

Again, the strategy sum does not depend upon the quality of information, and is equal to the coefficient multiplying the fundamental in the expression for the benchmark equilibrium quantity, so that the strategy distortion is at work, with its effects derived when information is perfect.

As to the relative weight on the public signal, we have

$$
\frac{\zeta^{Q}}{\zeta^{Q}+\xi^{Q}}=\frac{(1+\gamma) \alpha / \beta}{(1+\gamma) \alpha / \beta+1+n \gamma-(n-1) \gamma \lambda / 2}=\kappa^{Q}(\lambda) .
$$

Its efficient value $\kappa^{Q}(0)$ is again increasing in the level of interdependence $(n-1)|\gamma|$ under the coordination motive (now when $\gamma<0$ ), hence under strategic complementarity. It is decreasing under the anti-coordination motive (when $\gamma>0$ ), hence under strategic substitutability. The competition motive induces an informational distortion, by decreasing (increasing) $\kappa^{Q}(\lambda)$ under the coordination (anti-coordination) motive, hence by countervailing the effect of this motive. In relative terms,

$$
\frac{\left|\kappa^{Q}(0)-\kappa^{Q}(\lambda)\right|}{\kappa^{Q}(0)}=\frac{(n-1)|\gamma| \lambda / 2}{(1+\gamma) \alpha / \beta+1+n \gamma-(n-1) \gamma \lambda / 2},
$$

increasing in $(n-1)|\gamma| \lambda$ and decreasing in $\alpha / \beta$.

To recall,

Proposition 4 Under imperfect and dispersed information, the competition motive generates, in both price and quantity games, an informational distortion which countervails the effect of the coordination (anti-coordination) motive, making the relative weight put on the public signal inefficiently low (high) under strategic complementarity (substitutability). This distortion is the stronger the higher the intensity of competition $(n-1)|\gamma| \lambda$ and the lower the relative precision $\alpha / \beta$ of the public signal. 


\subsection{Welfare}

Profit, social welfare and consumer surplus are defined as in the benchmark case, but we must now refer to their mathematical expectations, conditional on the realized $\theta$.

- The price game

The expected profit is

$$
\mathbb{E}\left(\Pi^{P} \mid \theta\right)=\frac{1}{1+n \gamma} \mathbb{E}\left(2 \theta \sum_{i} p_{i}^{*}-(1+n \gamma) \sum_{i} p_{i}^{* 2}+\gamma\left(\sum_{i} p_{i}^{*}\right)^{2} \mid \theta\right),
$$

with $p_{i}^{*}=K^{P}(\lambda)\left(\theta+\kappa^{P}(\lambda) \eta+\left(1-\kappa^{P}(\lambda)\right) \varepsilon_{i}\right)$, hence equal to

$$
\begin{aligned}
& \mathbb{E}\left(\Pi^{P} \mid \theta\right)=\frac{n\left(K^{P}(\lambda)\right)^{2}}{1+n \gamma}\left[\left(\frac{2}{K^{P}(\lambda)}-1\right) \theta^{2}-C^{P}\right], \\
& \text { where } C^{P}=\frac{\left(\kappa^{P}(\lambda)\right)^{2}}{\alpha}+(1+(n-1) \gamma) \frac{\left(1-\kappa^{P}(\lambda)\right)^{2}}{\beta}
\end{aligned}
$$

is an information cost, the only element in the expression of $\mathbb{E}\left(\Pi^{P} \mid \theta\right)$ which depends upon information use and quality. Naturally, taking $C^{P}=0$, the expected profit is the one obtained under perfect information. The information cost is minimized in $\kappa$ under the first order condition:

$$
\frac{\kappa}{1-\kappa}=(1+(n-1) \gamma) \frac{\alpha}{\beta}=\frac{\kappa^{P}(0)}{1-\kappa^{P}(0)} \text {. }
$$

We see that the efficient use of information by the company corresponds indeed to $\lambda=0$ : no competition motive and no resulting informational distortion.

The expected social welfare is

$$
\begin{aligned}
\mathbb{E}\left(u^{P} \mid \theta\right) & =\mathbb{E}\left(2 \theta \sum_{i} Q_{i}\left(\mathbf{p}^{*}\right)-\frac{1}{2} \sum_{i}\left(Q_{i}\left(\mathbf{p}^{*}\right)\right)^{2}-\frac{\gamma}{2}\left(\sum_{i} Q_{i}\left(\mathbf{p}^{*}\right)\right)^{2} \mid \theta\right), \\
\text { with } Q_{i}\left(\mathbf{p}^{*}\right) & =\frac{1}{1+n \gamma}\left(\begin{array}{c}
2 \theta-K^{P}(\lambda)\left(\theta+\kappa^{P}(\lambda) \eta\right) \\
-K^{P}(\lambda)\left(1-\kappa^{P}(\lambda)\right)\left((1+n \gamma) \varepsilon_{i}-\gamma \sum_{j} \varepsilon_{j}\right)
\end{array}\right),
\end{aligned}
$$

hence

$$
\mathbb{E}\left(u^{P} \mid \theta\right)=\frac{n\left(K^{P}(\lambda)\right)^{2}}{2(1+n \gamma)}\left[\left(\frac{4}{\left(K^{P}(\lambda)\right)^{2}}-1\right) \theta^{2}-C^{P}\right] .
$$

Again, taking $C^{P}=0$, the expected social welfare is the one obtained under perfect information. By comparing (19) and (20), we see that the efficient use of information by the company, minimizing the information cost $C^{P}$ and prevailing in the absence of the competition motive, is socially optimal too. 
Finally, the expected consumer surplus is

$$
\mathbb{E}\left(u^{P} \mid \theta\right)-\mathbb{E}\left(\Pi^{P} \mid \theta\right)=\frac{n\left(K^{P}(\lambda)\right)^{2}}{2(1+n \gamma)}\left[\left(\frac{2}{K^{P}(\lambda)}-1\right)^{2} \theta^{2}+C^{P}\right] .
$$

Maximization in $\kappa$ of the consumer surplus would now require maximization of the information cost, constrained by equilibrium implementation, which can only result from the corner solution $\lambda=1$, as $C^{P}$ is a strictly convex function of $\kappa$.

- The quantity game

The expected profit is now

$$
\mathbb{E}\left(\Pi^{Q} \mid \theta\right)=\mathbb{E}\left(2 \theta \sum_{i} q_{i}^{*}-\sum_{i} q_{i}^{* 2}-\gamma\left(\sum_{i} q_{i}^{*}\right)^{2} \mid \theta\right),
$$

with $q_{i}^{*}=K^{Q}(\lambda)\left(\theta+\kappa^{Q}(\lambda) \eta+\left(1-\kappa^{Q}(\lambda)\right) \varepsilon_{i}\right)$, hence equal to

$$
\begin{aligned}
\mathbb{E}\left(\Pi^{Q} \mid \theta\right) & =n(1+n \gamma)\left(K^{Q}(\lambda)\right)^{2}\left[\left(\frac{2}{(1+n \gamma) K^{Q}(\lambda)}-1\right) \theta^{2}-C^{Q}\right](21) \\
\text { where } C^{Q} & =\frac{\left(\kappa^{Q}(\lambda)\right)^{2}}{\alpha}+\frac{1+\gamma}{1+n \gamma} \frac{\left(1-\kappa^{Q}(\lambda)\right)^{2}}{\beta}
\end{aligned}
$$

is the information cost $C^{Q}$, which is the only component depending upon information use and quality, and which is minimized in $\kappa$ under the first order condition:

$$
\frac{\kappa}{1-\kappa}=\frac{1+\gamma}{1+n \gamma} \frac{\alpha}{\beta}=\frac{\kappa^{Q}(0)}{1-\kappa^{Q}(0)},
$$

with no competition motive and no resulting informational distortion, a situation corresponding to the efficient use of information by the company.

The expected social welfare is

$$
\begin{aligned}
\mathbb{E}\left(u^{Q} \mid \theta\right) & =\mathbb{E}\left(2 \theta \sum_{i} q_{i}{ }^{*}-\frac{1}{2} \sum_{i} q_{i}{ }^{* 2}-\frac{\gamma}{2}\left(\sum_{i} q_{i}{ }^{*}\right)^{2} \mid \theta\right) \\
& =\frac{n(1+n \gamma)\left(K^{Q}(\lambda)\right)^{2}}{2}\left[\left(\frac{4}{(1+n \gamma) K^{Q}(\lambda)}-1\right) \theta^{2}-C^{Q}\right],
\end{aligned}
$$

with the sole term $C^{Q}$ depending on information use and quality. The efficient use of information by the company is consequently socially optimal.

As to the expected consumer surplus, it is equal to

$$
\mathbb{E}\left(u^{Q} \mid \theta\right)-\mathbb{E}\left(\Pi^{Q} \mid \theta\right)=\frac{n(1+n \gamma)\left(K^{Q}(\lambda)\right)^{2}}{2}\left(\theta^{2}+C^{Q}\right) .
$$


Since the information cost, the sole term depending upon information use and quality, appears now with an opposite sign, the expected consumer surplus would be maximized under a maximum strength $\lambda=1$ of the competition motive.

We summarize the preceding results of this subsection in the following proposition.

Proposition 5 Under imperfect and dispersed information, the information cost components of expected profit and social welfare are minimized in $\kappa$ at $\kappa^{P}(0)$ and $\kappa^{Q}(0)$ for the price and quantity games, respectively: efficient and socially optimal uses of information always coincide, prevailing when the competition motive is suppressed. By contrast, the consumer surplus is maximized in $\kappa$, under the constraint of equilibrium implementation, when the competition motive reaches its maximum strength.

\subsection{The social value of information}

Information quality can only influence the mathematical expectations of profit, social welfare and consumer surplus through the cost of information, $C^{P}$ or $C^{Q}$ according to the type of the game. Now, an increase in the information quality (a higher precision $\alpha$ or $\beta$ ) does not necessarily diminish the information cost because of the possible countervailing effect of a less efficient use of information due to the informational distortion. Let us consider the two types of game, successively.

- The price game

Recall that, according to equation (19), the information cost is

$$
C^{P}=\frac{\left(\kappa^{P}(\lambda)\right)^{2}}{\alpha}+(1+(n-1) \gamma) \frac{\left(1-\kappa^{P}(\lambda)\right)^{2}}{\beta} .
$$

As $\left(\partial \kappa^{P}(\lambda) / \partial(\alpha / \beta)\right) \alpha / \beta=\left(1-\kappa^{P}(\lambda)\right) \kappa^{P}(\lambda)$, we have

$$
\begin{aligned}
\frac{\partial C^{P}}{\partial \alpha} & =-\frac{\left(\kappa^{P}(\lambda)\right)^{2}}{\alpha^{2}}\left(1-\alpha \frac{\partial C^{P}}{\partial \kappa} \frac{1-\kappa^{P}(\lambda)}{\kappa^{P}(\lambda)}\right) \text { and } \\
\frac{\partial C^{P}}{\partial \beta} & =-\frac{\left(1-\kappa^{P}(\lambda)\right)^{2}}{\beta^{2}}\left(1+\beta \frac{\partial C^{P}}{\partial \kappa} \frac{\kappa^{P}(\lambda)}{1-\kappa^{P}(\lambda)}\right),
\end{aligned}
$$

so that the information cost unequivocally decreases in response to a higher precision $\alpha$ or $\beta$, as long as the competition motive is inactive $(\lambda=0)$, entailing $\partial C^{P} / \partial \kappa=0$ by optimality of $\kappa^{P}(0)$. Under the coordination motive (when $\gamma>0), \kappa^{P}(\lambda)<\kappa^{P}(0)$ for $\lambda>0$, so that $\partial C^{P} / \partial \kappa<0$ by strict convexity of $C^{P}$ relative to $\kappa$. The information cost is then decreasing in the precision $\alpha$ of the public signal, but not necessarily in the precision $\beta$ of the private signal, a result reversing the main conclusion of Morris and Shin (2002), obtained in a 
situation where social welfare depends upon the sole fundamental motive, given the existence of an exact balance between the coordination and competition motives. ${ }^{12}$

To check that the information cost may increase with more precise private information, consider the term

$$
\beta \frac{\partial C^{P}}{\partial \kappa} \frac{\kappa^{P}(\lambda)}{1-\kappa^{P}(\lambda)}=-\frac{(1+(n-1) \gamma)(n-1) \gamma \lambda}{1+(n-1) \gamma \lambda / 2} \kappa^{P}(\lambda),
$$

which is smaller than -1 , entailing positivity of $\partial C^{P} / \partial \beta$, for a high enough intensity of competition (with $\gamma>0$ ). This result contradicts Corollary 11 in Angeletos and Pavan (2007), which excludes in a similar context the case of a profit depressing effect of a higher precision of the private signal. Appendix A.2 offers a direct comparison between our result and theirs. It emphasizes in particular the fact that in the specification of Angeletos and Pavan, one can obtain a decrease in expected profits as the precision of the private signal increases (contrary to what is stated in Corollary 11), a possibility open for a high enough intensity of competition and a high enough precision of the public signal relative to that of the private signal, provided the slope of the marginal cost curve is itself large enough (entailing a high price response to demand shocks). In our own specification, without production costs, such possibility is consequently excluded with the information structure postulated by Angeletos and Pavan, that is, assuming prior public information on the stochastic fundamental $\theta$. However, in the absence of prior information, the role of the posterior public signal is reinforced, opening again the possibility that more precision of the private signal decreases expected profits as competition becomes sufficiently intense.

Under the anti-coordination motive (when $\gamma<0), \kappa^{P}(\lambda)>\kappa^{P}(0)$, hence $\partial C^{P} / \partial \kappa>0$, so that $\partial C^{P} / \partial \beta$ is always negative. So is $\partial C^{P} / \partial \alpha$, since

$$
\alpha \frac{\partial C^{P}}{\partial \kappa} \frac{1-\kappa^{P}(\lambda)}{\kappa^{P}(\lambda)}=-(n-1) \gamma \lambda\left(1-\kappa^{P}(\lambda)\right)<(1-1 / n) \lambda\left(1-\kappa^{P}(\lambda)\right)<1 .
$$

- The quantity game

By equation (21), the information cost is

$$
C^{Q}=\frac{\left(\kappa^{Q}(\lambda)\right)^{2}}{\alpha}+\frac{1+\gamma}{1+n \gamma} \frac{\left(1-\kappa^{Q}(\lambda)\right)^{2}}{\beta} .
$$

\footnotetext{
${ }^{12}$ See footnote 8 . As the distorted relative weight $\kappa(\lambda)$ on the public signal belongs to the interval $[\alpha /(\alpha+\beta), \kappa(0)]$, the reversal of results is the natural consequence of referring to an optimal $\alpha /(\alpha+\beta)$, as in Morris and Shin (2002) instead of an optimal $\kappa(0)$, as in the present context. See also Angeletos and Pavan (2007, p. 1126) commenting on such reversal obtained in other contexts.
} 
As $\left(\partial \kappa^{Q}(\lambda) / \partial(\alpha / \beta)\right) \alpha / \beta=\left(1-\kappa^{Q}(\lambda)\right) \kappa^{Q}(\lambda)$, we have

$$
\begin{aligned}
\frac{\partial C^{Q}}{\partial \alpha} & =-\frac{\left(\kappa^{Q}(\lambda)\right)^{2}}{\alpha^{2}}\left(1-\alpha \frac{\partial C^{Q}}{\partial \kappa} \frac{1-\kappa^{Q}(\lambda)}{\kappa^{Q}(\lambda)}\right) \\
\frac{\partial C^{Q}}{\partial \beta} & =-\frac{\left(1-\kappa^{Q}(\lambda)\right)^{2}}{\beta^{2}}\left(1+\beta \frac{\partial C^{Q}}{\partial \kappa} \frac{\kappa^{Q}(\lambda)}{1-\kappa^{Q}(\lambda)}\right) .
\end{aligned}
$$

As in the price game, the information cost unequivocally decreases in response to a higher precision $\alpha$ or $\beta$, as long as the competition motive is inactive $(\lambda=0)$, entailing $\partial C^{Q} / \partial \kappa=0$ by optimality of $\kappa^{Q}(0)$. Under the anti-coordination motive (when $\gamma>0$ ), $\kappa^{Q}(\lambda)>\kappa^{Q}(0)$ for $\lambda>0$, so that $\partial C^{Q} / \partial \kappa>0$ by strict convexity of $C^{Q}$ relative to $\kappa$. The information cost is then always decreasing in the precisions $\alpha$ and $\beta$ of both signals, since $\partial C^{Q} / \partial \beta$ is clearly negative, and so is $\partial C^{Q} / \partial \alpha$, because

$$
\alpha \frac{\partial C^{Q}}{\partial \kappa} \frac{1-\kappa^{Q}(\lambda)}{\kappa^{Q}(\lambda)}=\frac{(n-1) \gamma \lambda}{1+n \gamma}\left(1-\kappa^{Q}(\lambda)\right)<1 .
$$

This result differs from that stated in Corollary 10 of Angeletos and Pavan (2007), admitting that the expected profit can decrease with the precision of the public signal. The reason for this difference is formally explained in Appendix A.1. As already emphasized for the price game, our information structure differs slightly from theirs, as we consider no prior public information. The presence of prior information limits the role of the public signal received a posteriori by the players. By contrast, in the absence of such prior information, making its variance tend to infinity in the specification of Angeletos and Pavan, the role of the public signal is reinforced, and more precision of this signal always translates into higher expected profits, even when competition is intense.

Under the coordination motive (when $\gamma<0$ ), $\partial C^{Q} / \partial \kappa<0$, hence $\partial C^{Q} / \partial \alpha<$ 0 , but $\partial C^{Q} / \partial \beta$ may be positive as

$$
\beta \frac{\partial C^{Q}}{\partial \kappa} \frac{\kappa^{Q}(\lambda)}{1-\kappa^{Q}(\lambda)}=\frac{1+\gamma}{1+n \gamma} \frac{(n-1) \gamma \lambda}{1+n \gamma-(n-1) \gamma \lambda / 2} \kappa^{Q}(\lambda)<-1
$$

for a high enough intensity of competition (as $n \gamma \rightarrow-1$ ).

We may now summarize the preceding results of this subsection in the following proposition.

Proposition 6 Variations of information quality have, in both games, the same qualitative effects on expected profit and social welfare, and opposite effects on expected consumer surplus. In the absence of the competition motive or else under the anti-coordination motive, both precisions are always profit and welfare enhancing: information has necessarily a positive social value. However, under the coordination motive, hence under strategic complementarity, if profit and social welfare always increase with a higher precision $\alpha$ of the public signal, they decrease, for a high enough intensity of competition, when the precision $\beta$ of the private signal increases. 
A few comments on the reasons for the results stated in this proposition are in order. An increase in the precision of any of the two signals has a favorable direct effect on the information cost, hence on profit and welfare, plus an indirect effect (if $\lambda>0$ ) through the relative weight $\kappa(\lambda)$ (see equations (23)-(24) for the price game and (26)-(27) for the quantity game). Under the coordination motive, hence under strategic complementarity, $\kappa(\lambda)<\kappa(0)$ : the informational distortion leads to an excessive weight put on the private signal. As this distortion is decreasing in the ratio of precisions $\alpha / \beta$, an increase in the quality of public - not private - information (see (25) for the price game and (28) for the quantity game) has a favorable indirect effect on the information cost. So, we are left with two opposite effects of an increase in the precision of the private signal. As the intensity of competition hence the informational distortion increases, the unfavorable indirect effect will eventually dominate. The analysis is not completely symmetric when we switch to the case of the anti-coordination motive, a situation in which the informational distortion leads to an excessive weight put on the public signal. This is because indefinitely increasing the intensity of competition keeps the anti-coordination and competition motives dominated by the fundamental motive. As a consequence, the favorable direct effects always dominate.

\subsection{Price vs. quantity games}

In this subsection we compare price and quantity games from a welfare perspective. Take first, using equations (19) and (21), the difference in expected profits obtained when playing each kind of strategies:

$$
\begin{aligned}
& \mathbb{E}\left(\Pi^{Q} \mid \theta\right)-\mathbb{E}\left(\Pi^{P} \mid \theta\right) \\
= & \frac{n}{1+n \gamma}\left[\begin{array}{c}
\left(\left(1-K^{P}(\lambda)\right)^{2}-\left((1+n \gamma) K^{Q}(\lambda)-1\right)^{2}\right) \theta^{2} \\
+\left(K^{P}(\lambda)\right)^{2} C^{P}-\left((1+n \gamma) K^{Q}(\lambda)\right)^{2} C^{Q}
\end{array}\right] .
\end{aligned}
$$

The first term in the brackets is the difference found in the benchmark perfect information case, corresponding here to $C^{P}=C^{Q}=0$, due either to $\alpha=\infty$ or to $\beta=\infty$. We know that, when the goods are independent $(\gamma=0)$ or when the competition motive is absent $(\lambda=0), K^{P}=(1+n \gamma) K^{Q}=1$, so that we are left with the difference $C^{P}-C^{Q}$ to determine the sign of this expression. If $\gamma=0, C^{P}=C^{Q}=1 /(\alpha+\beta)$, so that it is indifferent to play in prices or in quantities in the case of independence of goods. If $\lambda=0$, the same is true, provided information, if not perfect, is at least homogeneous $(\beta=0)$, since we have then $C^{P}=C^{Q}=1 / \alpha$.

Now, still with $\lambda=0$ but with $\alpha<\infty$ and $0<\beta<\infty$, it is straightforward to check that the difference

$$
C^{P}-C^{Q}=\frac{(2+n \gamma)(n-1) \beta \gamma}{[(1+\gamma) \alpha+(1+n \gamma) \beta][(1+(n-1) \gamma) \alpha+\beta]}
$$

has the sign of $\gamma$ : playing under the anti-coordination motive (hence under strategic substitutability) is better for the company. Information costs alone 
(that is, when $\lambda=0$, leading to equal weights on $C^{P}$ and $C^{Q}$ ) leads to the same result as the one induced by the strategy distortion in the benchmark situation of perfect information. However, as the intensity of competition increases, the two combined distortions start to exert opposite effects since the relative weight put on the higher information cost becomes lower and lower. The previous result may then be reversed. Indeed, when goods are substitutes $(\gamma>0)$, as the intensity of competition tends to infinity, the weight $\left(K^{P}(\lambda)\right)^{2}$ put on $C^{P}$ tends to zero, so that the negative term involving $C^{Q}$ on the RHS of equation (29) eventually becomes the dominant term for a large enough $C^{Q}$ (for small enough precisions of the two signals). If the goods are complements $(\gamma<0)$, as the intensity of competition tends to its upper bound $(1-1 / n) / 2$, the weight $(1+n \gamma) K^{Q}(\lambda)$ put on $C^{Q}$ tends to zero, so that again the result may be reversed for a large enough $C^{P}$ (for low enough precisions of the two signals). In other words, poor information quality and high intensity of competition can result in the company preferring the game where the players obey to a coordination motive, under strategic complementarity.

As to the expected social welfare, we use equations (20) and (22) and compute:

$\mathbb{E}\left(u^{Q} \mid \theta\right)-\mathbb{E}\left(u^{P} \mid \theta\right)=\frac{n}{2(1+n \gamma)}\left[\begin{array}{c}\left(\left(K^{P}(\lambda)\right)^{2}-\left(2-(1+n \gamma) K^{Q}(\lambda)\right)^{2}\right) \theta^{2} \\ +\left(K^{P}(\lambda)\right)^{2} C^{P}-\left((1+n \gamma) K^{Q}(\lambda)\right)^{2} C^{Q}\end{array}\right]$.

The first term in the brackets corresponds to the difference we have found in the perfect information benchmark case (see equation (17)). It is always negative, thus introducing a bias in favor of the game in prices, due to the presence of the strategy distortion. The second term is identical to the corresponding term in equation (29), for the difference between the profits obtained in the two games. Thus, the results expressing the point of view of the company apply to social welfare.

Finally, from the point of view of the consumer, we have:

$$
\begin{gathered}
\left(\mathbb{E}\left(u^{Q} \mid \theta\right)-\mathbb{E}\left(\Pi^{Q} \mid \theta\right)\right)-\left(\mathbb{E}\left(u^{P} \mid \theta\right)-\mathbb{E}\left(\Pi^{P} \mid \theta\right)\right) \\
=\frac{n}{2(1+n \gamma)}\left[\begin{array}{c}
\left(\left((1+n \gamma) K^{Q}(\lambda)\right)^{2}-\left(2-K^{P}(\lambda)\right)^{2}\right) \theta^{2} \\
-\left(K^{P}(\lambda)\right)^{2} C^{P}+\left((1+n \gamma) K^{Q}(\lambda)\right)^{2} C^{Q}
\end{array}\right] .
\end{gathered}
$$

Naturally, the first term between brackets is the same as in the benchmark case, and the second term is equal, but with an opposite sign, to the corresponding term for the difference in social welfare and, up to a factor $1 / 2$, for the difference in profits.

We can now summarize the preceding results in the following proposition.

Proposition 7 The pure presence of information costs, in the absence of the competition motive, favors anti-coordination from the point of view of the company (hence, the quantity game when goods are substitutes and the price game when they are complements). However, as the intensity of competition increases, 
this effect is reinforced by the strategy distortion, but is countervailed by the informational distortion, which eventually reverses the result under poor information quality. The informational distortion has the same influence on social welfare, in spite of having an opposite influence on the consumer surplus.

\section{Conclusion}

We proposed a simple unified framework bridging the gap between team and competition issues under imperfect and dispersed information, and allowing to reconsider the social value of information in price and quantity games and to compare their outcomes in terms of equilibrium and social welfare.

The competition motive creates, even when information is perfect, a wellknown strategy distortion and, in addition, when information is imperfect and dispersed, an informational distortion. Both distortions are detrimental to profitability, and the more so the higher the intensity of competition, through either structure (by the degree of interdependence between goods or by the number of competitors) or conduct (by the strength of the competition motive). The informational distortion generated by the competition motive in both price and quantity games is detrimental as it makes the relative weight put on the public signal inefficiently high (low) under the anti-coordination (coordination) motive, hence under strategic substitutability (complementarity). Both distortions interact and the dominance of one over the other depends on the information precision and naturally lead to different effects in terms of equilibrium and social welfare.

Regarding the social value of information, we show that increasing the precision of public information always improves welfare. By contrast, the social value of private information may be negative under the coordination motive (in the presence of strategic complementarity) for a high enough intensity of competition.

While the competition motive operating under anti-coordination (in the presence of strategic substitutability) yields higher profit and social welfare when information is perfect, imperfect and dispersed information mitigates such a result. Indeed, if the intensity of competition is sufficiently strong and information quality is relatively low, making the information distortion dominate the strategy distortion, profit and social welfare are higher under coordination (in the presence of strategic complementarity). The reverse is true from the consumers' point of view.

Finally, we may derive some policy implications from our findings. First, while the results obtained earlier in the literature (e.g. Gal-Or, 1986, p. 91) indicate that an industry has some incentives to "create an 'association' that collects and publicizes information depend[ing] upon the type of competition in the industry and the source of uncertainty in the market", we argue that such an association is relevant independently from the type of competition. Though beneficial from the firms' point of view and with regard to the overall welfare, such an association is however detrimental in the consumers' perspective. 
Second, our results may have some implications on the performance of the competition regimes (price vs. quantity competition). In a context where the company divisions have poorly accurate information on the fundamental (the market size), they may prefer to compete in prices (quantities) if goods are substitutes (complements). A regulator that would increase the precision of public information might reverse such preference by reducing the weight of the informational distortion relative to that of the strategy distortion. Again, this would however be at the expense of the consumers.

The restriction of the analysis performed in this paper to symmetric games and symmetric solutions is of course a limitation that we intend to remove in further research. As structural asymmetries have already been dealt with in the related context of firm-specific information sharing in oligopolies (Amir et al., 2010), we think in particular of the conduct asymmetry regarding the strength of the competition motive assigned to each division (or adopted by each firm). How does this asymmetry impend upon the social value of public information? Also, allowing for asymmetry in the precision of the private signals, how does an improvement in the quality of private information benefitting to some particular firm impact on its own profit and on the profit of its competitors? Are these effects modulated by the corresponding strengths of the competition motive? These are typical questions we want to address in the future.

\section{References}

Amir, R., Erickson, P. and Jin, J. (2017). On the microeconomic foundations of linear demand for differentiated products. Journal of Economic Theory, 169: 641-665.

Amir, R. and Jin, J. (2001). Cournot and Bertrand equilibria compared: substitutability, complementarity and concavity. International Journal of Industrial Organization, 19(3-4): 303-317.

Amir, R., Jin, J., Pech, G. and Troege, M. (2016). Prices and Deadweight Loss in Multi-Product Monopoly. Journal of Public Economics Theory, 18: 346-362.

Amir, R., Jin, J. and Troege, M. (2010). Robust Results on the Sharing of FirmSpecific Information: Incentive and Welfare Effects. Journal of Mathematical Economics, 46: 855-866.

Angeletos, G.-M. and Pavan, A. (2007). Efficient use of information and social value of information. Econometrica, 75: 1103-1142.

Cornand, C. and Dos Santos Ferreira, R. (2017). Cooperation in a differentiated duopoly when information is dispersed: A beauty contest game with endogenous concern for coordination, Working Paper BETA 2017-05.

Cornand, C. and Heinemann, F. (2008). Optimal degree of public information dissemination. Economic Journal, 118: 718-742.

Cournot, A. (1838). Recherches sur les principes mathématiques de la théorie 
des richesses. English translation by Nathaniel T. Bacon: Researches into the Mathematical Principles of the Theory of Wealth, New York: Macmillan, 1897.

Cremer, J. (1990). Common knowledge and the coordination of economic activities. in M. Aoki, B. Gustafsson, and O. E. Williamson, eds, The Firm as a Nexus of Treaties, 53-76, London: Sage Publishers.

Gardete, P. (2016). Competing Under Asymmetric Information: The case of dynamic random access memory manufacturing. Management Science, 62: 32913309 .

Gal-Or, E. (1985). Information sharing in oligopoly. Econometrica, 53: 329-343.

Gal-Or, E. (1986). Information transmission - Cournot and Bertrand equilibria. Review of Economic Studies, 53: 85-92.

Li, L. (1985). Cournot oligopoly with information sharing. RAND Journal of Economics, 16: 521-536.

Mahoney, J. (1992). The adoption of the multidivisional form of organization: A contingency model. Journal of Management Studies, 29: 49-72.

Morris, S. and Shin, H. S. (2002). Social value of public information. American Economic Review, 92: 1521-1534.

Myatt, D. P. and Wallace, C. (2012). Endogenous information acquisition in coordination games. Review of Economic Studies, 79: 340-374.

Myatt, D. P. and Wallace, C. (2015). Cournot competition and the social value of information. Journal of Economic Theory, 158: 466-506.

Myatt, D. P. and Wallace, C. (2018). Information use and acquisition in pricesetting oligopolies. Economic Journal, 128: 845-886.

Novshek, W. and Sonnenschein, H. (1982). Fulfilled expectations Cournot duopoly with information acquisition and release. Bell Journal of Economics, 13: 214-218.

Radner, R. (1962). Team decision problems. Annals of Mathematical Statistics, 33: $857-881$.

Raith, M. (1996). A general model of information sharing in oligopoly. Journal of Economic Theory, 71: 260-288.

Sakai, Y. (1985). The value of information in a simple duopoly model. Journal of Economic Theory, 36: 36-54.

Singh, N. and Vives, X. (1984). Price and quantity competition in a differentiated duopoly. RAND Journal of Economics, 15: 546-554.

Ui, T. (2009). Bayesian potentials and information structures: Team decision problems revisited. International Journal of Economic Theory, 5: 271-291.

Ui, T. and Yoshizawa, Y. (2015). Characterizing social value of information. 
Journal of Economic Theory, 158: 507-535.

Vives, X. (1984). Duopoly information equilibrium: Cournot and Bertrand. Journal of Economic Theory, 34: 71-94.

Vives, X. (1988). Aggregation of information in large Cournot markets. Econometrica, 56: 851-876.

Vives, X. (2008), Information sharing among firms, The New Palgrave Dictionary of Economics, S. N. Durlauf and L. E. Blume (eds.), Second Edition.

Vives, X. (2011). Strategic supply function competition with private information. Econometrica, 79: 1919-1966.

\section{A The welfare effects of changes in the precision of public and private information: A compar- ison with Angeletos and Pavan $(2007,6.5)$}

In Angeletos and Pavan (2007) (henceforth AP), payoffs depend upon a stochastic fundamental, which may be expressed as $\theta=\mu+\tau$, where $\mu$ is constant and $\tau$ is normally distributed with zero mean and variance $\sigma_{\theta}^{2}$. The realization $\theta$ of the fundamental cannot be observed by the players, who receive instead a public signal $y=\theta+\eta$ and private signals $x_{i}=\theta+\varepsilon_{i}$. The noises of the common and idiosyncratic signals are normally distributed with zero mean and variances $\sigma_{y}^{2}$ and $\sigma_{x}^{2}$, respectively, and are uncorrelated between each other, as well as with other private signals and the fundamental. In the following, it will be sometimes convenient to refer directly to the synthetic public signal $z \equiv \mathbb{E}(\theta \mid y)=$ $\phi y+(1-\phi) \mu$, where $\phi \equiv \sigma_{y}^{-2} /\left(\sigma_{y}^{-2}+\sigma_{\theta}^{-2}\right)$. The public signal $z$ is itself normally distributed with mean $\mu$ and variance $\sigma_{z}^{2}=1 /\left(\sigma_{y}^{-2}+\sigma_{\theta}^{-2}\right) \equiv 1 / \alpha$. The private posterior is also normal with mean $\mathbb{E}\left(\theta \mid x_{i}, y\right)=\left(\alpha z+\beta x_{i}\right) /(\alpha+\beta)$, where $\beta \equiv \sigma_{x}^{-2}$, and variance $\operatorname{Var}\left(\theta \mid x_{i}, y\right)=1 /(\alpha+\beta)$.

In what follows, we show that in the Cournot game our result on the welfare enhancing effect of a higher precision of the public signal differs from that stated in Corollary 10 of AP, because of the difference in the assumed information structure (infinite variance $\sigma_{\theta}^{2}$ in our model against finite variance in AP). We further show that in the Bertrand game our result on the possibly welfare depressing effect of a higher precision of the private signal differs for the same reason from that stated in Corollary 11 by AP. We also show that AP's result is not ensured, even under the assumed information structure. We provide the details of the calculations for the Bertrand game only.

\section{A.1 Cournot game}

Following AP, the inverse demand faced by firm $i$, competing against a continuum of identical firms which produce imperfectly substitutable goods, is given by $p_{i}=a_{0}+a_{1} \theta-a_{2} q_{i}-a_{3} Q$, with $a_{0}, a_{1}, a_{2}, a_{3}>0$, where $p$ is the selling 
price, $q$ the quantity of good put by the firm in the market, $Q$ the average quantity put in the market by the competitors, and $\theta$ an exogenous demand shifter. Individual payoffs are given by $u_{i}=p_{i} q_{i}-c_{1} q_{i}-c_{2} q_{i}^{2}$, with $c_{1}, c_{2}>0$. Since $x_{i}=\mu+\tau+\varepsilon_{i}$ and $z=\phi y+(1-\phi) \mu=\mu+\phi \tau+\phi \eta$, the equilibrium quantity in this model is given by:

$$
q_{i}=A+B \mu+B(1-\kappa(1-\phi)) \tau+B(1-\kappa) \varepsilon_{i}+B \kappa \phi \eta
$$

with $A \equiv \frac{a_{0}-c_{1}}{2\left(a_{2}+c_{2}\right)+a_{3}}, B \equiv \frac{a_{1}}{2\left(a_{2}+c_{2}\right)+a_{3}}$ and $\kappa=\frac{2\left(a_{2}+c_{2}\right) \alpha}{2\left(a_{2}+c_{2}\right) \alpha+\left(2\left(a_{2}+c_{2}\right)+a_{3}\right) \beta}$. The expected profit before realization of $\theta$ can then be expressed as

$$
\begin{aligned}
\mathbb{E}\left(u_{i}\right)= & C+\left(a_{1}-\left(a_{2}+c_{2}+a_{3}\right) B(1-\kappa(1-\phi))\right)(1-\kappa(1-\phi)) B \sigma_{\theta}^{2} \\
& -\left(a_{2}+c_{2}\right) B^{2}(1-\kappa)^{2} \sigma_{x}^{2}-\left(a_{2}+c_{2}+a_{3}\right) B^{2} \kappa^{2} \phi^{2} \sigma_{y}^{2},
\end{aligned}
$$

where $C$ is constant in terms of the information parameters. The derivative of this expression with respect to the precision $\sigma_{y}^{-2}$ of the public signal $y$, which can be negative according to Corollary 10 of AP, is

$$
\begin{aligned}
\frac{\partial \mathbb{E}\left(u_{i}\right)}{\partial \sigma_{y}^{-2}} & =\left(a_{2}+c_{2}+a_{3}\right) B^{2} \kappa^{2} \underbrace{\left(\phi \sigma_{y}^{2}\right)^{2}}_{1 / \alpha^{2}}+\frac{\partial \mathbb{E}\left(u_{i}\right)}{\partial \kappa} \frac{\partial \kappa}{\partial \sigma_{y}^{-2}}+\frac{\partial \mathbb{E}\left(u_{i}\right)}{\partial \phi} \frac{\partial \phi}{\partial \sigma_{y}^{-2}} \\
& =\left[a_{2}+c_{2}-a_{3}(1-\kappa)\right] B^{2}\left(\frac{\kappa}{\alpha}\right)^{2}<0 \text { only if } a_{2}+c_{2}<a_{3} .
\end{aligned}
$$

The latter necessary condition translates into $\gamma>1 /(n-2)$ in the model of our paper, where $a_{2}=1+\gamma, a_{3}=(n-1) \gamma$ and $c_{2}=0$, meaning intensive competition, due to high product substitutability and/or high number of competitors. However, with no a priori information on the fundamental $\left(\sigma_{\theta}^{2}=\infty\right.$, the case explored in our paper $), \phi=1$ and $\partial \phi / \partial \sigma_{y}^{-2}=0$, so that $\frac{\partial \mathbb{E}\left(u_{i}\right)}{\partial \sigma_{y}^{-2}}=\left[a_{2}+c_{2}+a_{3}+\left(a_{2}+c_{2}\right)(1-\kappa)\right] B^{2}\left(\frac{\kappa}{\alpha}\right)^{2}>0$ : a higher precision of the public signal always increases expected profits.

\section{A.2 Bertrand game}

Following AP, demand addressed to the individual firm $i$ is $q_{i}=b_{0}+b_{1} \theta^{\prime}-$ $b_{2} p_{i}+b_{3} P$, its payoff being again $u_{i}=p_{i} q_{i}-c_{1} q_{i}-c_{2} q_{i}^{2}$ or, simplifying notations, $u_{i}=b_{2}\left[k(\theta-k+b K)-c(\theta-k+b K)^{2}\right]$, with $k \equiv p_{i}-c_{1}, K \equiv P-c_{1}, \theta \equiv$ $\frac{b_{0}+b_{1} \theta^{\prime}-c_{1}\left(b_{2}-b_{3}\right)}{b_{2}}, b \equiv \frac{b_{3}}{b_{2}} \in(0,1)$, and $c \equiv c_{2} / b_{2}$. The best reply is determined by the first order condition for maximizing $u_{i}$ in $k$ :

$$
k=\frac{1+2 c}{2(1+c)} \mathbb{E}\left(\theta \mid x_{i}, y\right)+b \frac{1+2 c}{2(1+c)} \mathbb{E}\left(K \mid x_{i}, y\right) .
$$

Assuming linearity with respect to the received signals $\left(k_{i}=\delta_{1} x_{i}+\delta_{2} z\right)$, we have

$$
\begin{aligned}
k_{i} & =\delta_{1} x_{i}+\delta_{2} z \\
& =\frac{1+2 c}{2(1+c)} \frac{\alpha z+\beta x_{i}}{\alpha+\beta}+b \frac{1+2 c}{2(1+c)}\left(\delta_{1} \frac{\alpha z+\beta x_{i}}{\alpha+\beta}+\delta_{2} z\right),
\end{aligned}
$$


so that

$$
\begin{aligned}
\delta_{1} & =\frac{(1+2 c) \beta}{2(1+c) \alpha+(1+(1-b)(1+2 c)) \beta} \\
\delta_{2} & =\frac{2(1+c)}{2(1+c)-b(1+2 c)} \frac{(1+2 c) \alpha}{2(1+c) \alpha+(1+(1-b)(1+2 c)) \beta} \\
\delta_{1}+\delta_{2} & =\frac{1+2 c}{1+(1-b)(1+2 c)} \equiv B .
\end{aligned}
$$

We thus obtain:

$$
\begin{aligned}
k_{i} & =B\left(\begin{array}{c}
\overbrace{\frac{(1+(1-b)(1+2 c)) \beta}{2(1+c) \alpha+(1+(1-b)(1+2 c)) \beta}}^{1-\kappa} x_{i} \\
+\frac{2(1+c) \alpha}{\underbrace{2(1+c) \alpha+(1+(1-b)(1+2 c)) \beta}_{\kappa}} z
\end{array}\right) \\
& =B \mu+B(1-\kappa(1-\phi)) \tau+B(1-\kappa) \varepsilon_{i}+B \kappa \phi \eta .
\end{aligned}
$$

The expected profit is consequently

$$
\begin{aligned}
\mathbb{E}\left(u_{i}\right)= & b_{2} \mathbb{E}\left[k_{i}\left(\theta-k_{i}+b K\right)-c\left(\theta-k_{i}+b K\right)^{2}\right] \\
= & C+b_{2}\left(\begin{array}{c}
-c+B(1-\kappa(1-\phi))(1+2 c(1-b)) \\
-B^{2}(1-\kappa(1-\phi))^{2}(1-b)(1+c(1-b))
\end{array}\right) \sigma_{\theta}^{2} \\
& -b_{2}(1+c) B^{2}(1-\kappa)^{2} \sigma_{x}^{2}-b_{2}(1-b)(1+c(1-b)) B^{2} \kappa^{2} \phi^{2} \sigma_{y}^{2} .
\end{aligned}
$$

The derivative of this expression with respect to the precision $\sigma_{x}^{-2}$ of the private signal $x_{i}$, positive according to Corollary 11 of AP, is

$$
\frac{\partial \mathbb{E}\left(u_{i}\right)}{\partial \sigma_{x}^{-2}}=b_{2}(1+c) B^{2}\left(\frac{1-\kappa}{\beta}\right)^{2}+\frac{\partial \mathbb{E}\left(u_{i}\right)}{\partial \kappa} \frac{\partial \kappa}{\partial \sigma_{x}^{-2}},
$$

with

$$
\begin{aligned}
& \frac{\partial \mathbb{E}\left(u_{i}\right)}{\partial \kappa} \frac{\partial \kappa}{\partial \sigma_{x}^{-2}}=-\frac{\kappa(1-\kappa)}{\beta} \times \\
& {\left[\begin{array}{c}
b_{2}\left(\begin{array}{c}
-(1+2 c(1-b)) \\
+2 B(1-\kappa(1-\phi))(1-b)(1+c(1-b))
\end{array}\right) B \overbrace{(1-\phi) \sigma_{\theta}^{2}}^{1 / \alpha} \\
+2 b_{2}(1+c) B^{2}(1-\kappa) \underbrace{\sigma_{x}^{2}}_{1 / \beta}-2 b_{2}(1-b)(1+c(1-b)) B^{2} \kappa \phi \underbrace{\phi \sigma_{y}^{2}}_{1 / \alpha}
\end{array}\right],}
\end{aligned}
$$

so that

$$
\frac{\partial \mathbb{E}\left(u_{i}\right)}{\partial \sigma_{x}^{-2}}=\left[\begin{array}{c}
1 / 2+c(1-2 b) \\
+(1+2 c-2 B(1-b)(1+c(1-b)))(1-\kappa)
\end{array}\right] b_{2} B \frac{\kappa(1-\kappa)}{\alpha \beta} .
$$


The sign of $\partial \mathbb{E}\left[u_{i}\right] / \partial \sigma_{x}^{-2}$ can be negative, if $\kappa$ is close to 1 (if the precision of the public signal relative to the private one is very high) and if $b$ is itself close to 1 (if competition is very intense), provided $c>1 / 2$ (highly increasing marginal cost, magnifying the price response to a shock in the fundamental). This invalidates Corollary 11 of AP.

In our own specification, $c=0$, so that the sign of $\partial \mathbb{E}\left(u_{i}\right) / \partial \sigma_{x}^{-2}$ is always positive as long as $\sigma_{\theta}^{2}<\infty$. However, if we take the case of no a priori information, which implies $\phi=1$, the coefficient of $\sigma_{\theta}^{2}$ in $\mathbb{E}\left(u_{i}\right)$ ceases to depend upon $\kappa$ and becomes independent from the information parameters. As a consequence, we have

$$
\frac{\partial \mathbb{E}\left(u_{i}\right)}{\partial \sigma_{x}^{-2}}=[1 / 2+(1-b)(1 / 2+c)+((1-b)(1-2 b c)-1) \kappa] b_{2} B^{2} \frac{\kappa(1-\kappa)}{\alpha \beta},
$$

with the sign of $2-b(1+2 \kappa)$ for $c=0$. Again, this expression is negative if $b$ and $\kappa$ are both close enough to 1 . 